\title{
An Objective Image Quality Evaluation and Its Applications for Low Illumination and Sudden Illumination Changes
}

\author{
Thathupara Subramanyan Kavya, Young-Min Jang, Erdenetuya Tsogtbaatar, Tao Peng, Sang-Bock Cho \\ Department of Electrical Engineering, University of Ulsan, Republic of Korea.
}

ORCID: 0000-0001-8734-1980 (Young-Min Jang-second author)

\begin{abstract}
The various image-recording devices using a camera lens recognizes only the general surrounding environment. Further, it is difficult for a camera to recognize the images in a situation of sudden illumination change and low illumination. The various situation related to the dynamic illumination change interferes with recognition of the objects where object recognition is the prime requirement. In terms of traffic security application, the general image-recording devices cannot provide information about the traffic-related incidents due to illumination changes. The image quality evaluation methods such as luminance and contrast evaluation must take precedence to improve the image quality from sudden illumination changes and low illumination conditions. An objective image quality evaluation (OIQE) to assess the luminance and contrast of images is proposed in this paper. The proposed method uses each pixel's data as a weighted value of the probability density function. It is further defined by normalization for objective representation. The normalization technique always can display consistently irrespective of image resolution or change in the number of represented data bits. Also, it can be used in the adaptive gamma process because it suggests an objective basis of the gamma value selection. It is possible to use tone mapping to sudden illumination changes and low illumination conditions, because of its high dynamic range. As a result, the proposed technique can be incorporated with lane detection, number plate recognition, lens distortion correction, etc.
\end{abstract}

Keywords - Sudden illumination changes, low illumination, image quality, image contrast, image processing.

\section{INTRODUCTION}

In recent years, the use of the camera is increasing in various fields such as vehicle industry, mobile industry, CCTV etc. Moreover, the importance of image processing technology is growing rapidly. Most cameras capture the general surrounding environments when the conditions are ideal. However, when the conditions are non-ideal like low illumination, sudden change in illuminations etc, image capture become difficult. Some of the non-ideal situations are shown in Fig. 1. These non-ideal situations most commonly interferes with the recognition and capture of the objects using a camera. In the case of traffic security cameras, general image-recording devices cannot provide accurate information about the circumstances of the incidents.
When there are many light sources or low illumination conditions, the image sensor technology has to be utilized to get the exact information about the scenes [1][2][3][4]. But, the design and manufacturing of the image sensor have the disadvantage of enormous development cost and period. As an innovative method, the study of high dynamic range and gamma correction take centre stage in related fields. The image evaluation concerning luminance and contrast must take precedence to improve the image quality about sudden illumination change and low illumination conditions. But existing image evaluations and its study arbitrarily have interpreted about distribution of the histogram, and hence there is no way to present the criteria for objective evaluation [5][6][7].

The distribution of histogram was re-distributed by four areas of brightness and contrast, and the word descriptor that distribution density is expressed as a, A, b, B, c, C, d, D was introduced by Restrepo and Ramponi in 2008 [8]. However, this method is expressed as "very bright, very high contrast", objectivity and practicality of this method are too low. Therefore, to improve the objectivity, the word descriptor indicates a real number between zero and one in this paper, and we propose irrespective consistent normalization techniques of image resolution or change in several represented data bits. The proposed method suggests an objective basis to select the gamma value and hence can be used in the adaptive gamma process. Tone mapping can be used in sudden illumination changes and low illumination conditions due to its high dynamic range. As a result, the proposed techniques can be incorporated along with applications such as lane detection, number plate recognition, lens distortion correction, etc.
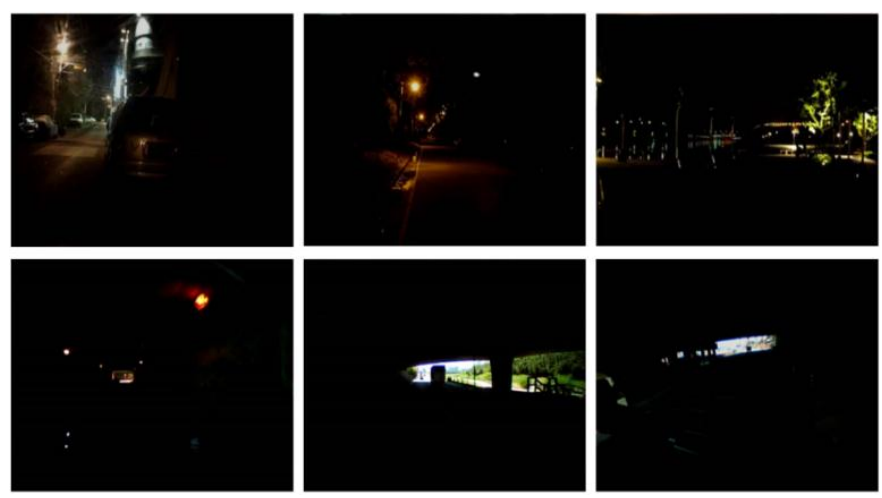

Fig. 1. Examples of low illumination and sudden illumination change. 


\section{RELATED WORK}

The graphical representation of the hue distribution of an image is the histogram. Variation of hue is represented in the horizontal axis and the number of pixels of the tone is represented in the vertical axis. A histogram of an image with a brightness $\mathrm{L}$ in range $([0, \mathrm{G}])$ is defined as $h\left(r_{\mathrm{k}}\right)=n_{\mathrm{k}}$. Where $r_{\mathrm{k}}$ is the $k$-th brightness level of range $([0, \mathrm{G}]), n_{\mathrm{k}}$ is the number of pixels with the brightness level of $r_{\mathrm{k}}$. If the image is 8 bits, the $G$ value is 255 and if the image if 16 bits, then the $\mathrm{G}$ value is 35535 . The RGB color model is an additive color model since the red, green and blue lights are added in different proportions to generate a broad range of colors. RGB color model is an array of color pixels of $\mathrm{M} \times \mathrm{N} \times 3$. RGB color cube representing the RGB color space is as shown in Fig. 2 (a). Another color space used by the imaging system is the $\mathrm{YCbCr}$ color model which is ss shown in Fig. 2 (b). In this model, $\mathrm{Cr}$ and $\mathrm{Cb}$ holds the color related information whereas the brightness information is represented by $\mathrm{Y}$ component. This is demonstrated as shown in Fig. 3. The $\mathrm{YCbCr}$ is a way of encoding RGB information and hence not an absolute color space. The actual color displayed is dependent on the RGB primary color of the display signal. A value expressed as $\mathrm{YCbCr}$ is predictable only if standard RGB chromaticities are used.

Image quality assessment has been studied by Oakley and $\mathrm{Bu}$. However, these image evaluations arbitrarily have been interpreted as the distribution of the histogram [9], and there is no way to present the criteria for objective evaluation. This method has the disadvantage of change, depending on main agents of the evaluation in a process that indicates the quality of the image as the density distribution of the value of the pixels data [10]. The pixel data of the image is divided into four regions (high contrast/middle luminance (A), low contrast/low luminance (B), middle contrast/middle luminance (C), low contrast/high luminance (D)) of $d$ - $l$ scatter chart by combined luminance and contrast which is introduced by Ramponid and Restrepo. For example, as shown in Fig. 4, by applying $Y=0.3$ to the image of a butterfly produce a bright image. Also, by applying $Y=2.5$ produce a dark image. These $d$ - $l$ scatter chart appears as a scatter plot shown on the bottom side of Fig. 4. As shown in equation (1), common distribution can be shown by maximum/minimum of data and sample from the group, including probability density function $(\mathrm{F})$ and cumulative distribution function (f).
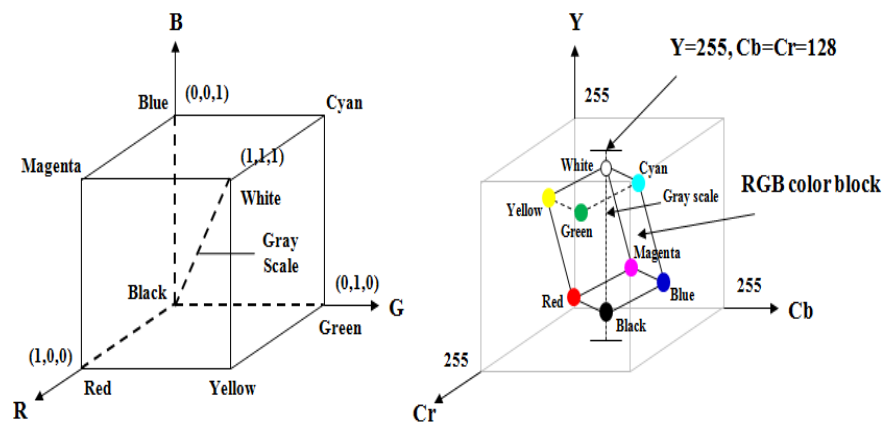

Fig. 2. The cube of (a) RGB color model (b) YCbCr color model

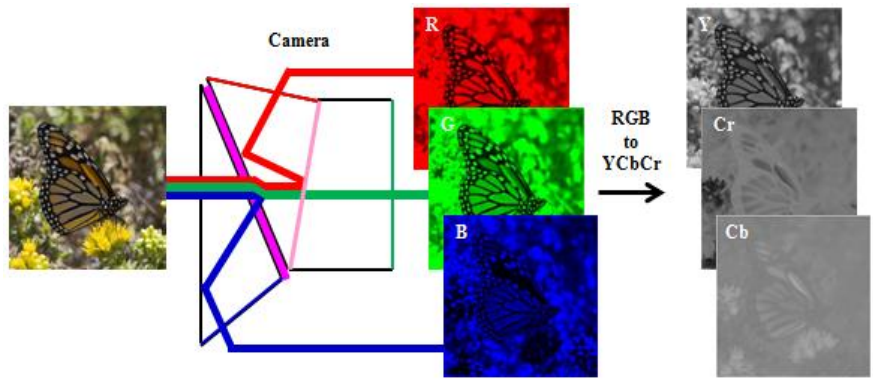

Fig. 3. The conversions to $\mathrm{YCbCr}$ model from RGB color model

$$
f_{\text {min,max }}(a, b)=n(n-1)[F(b)-F(a)]^{n-2} f(a) f(b)
$$

Also, common density function about uniform distribution with $\mathrm{F}(\mathrm{t})=1$ and $\mathrm{f}(\mathrm{t})=1$ can be shown by equation (2).

$$
\begin{aligned}
& f_{\text {min }, \max }(a, b)=n(n-1)[b-a]^{n-2} \\
& \quad a \in[0,1], a \leq b \leq 1
\end{aligned}
$$

where the maximum of $(\min , \max )$ is $(a, b)=(0,1)$ and the mathematical expectation value is given by equation (3).

$$
(E[\min ], E[\max ])=\left(\frac{1}{n+1}, \frac{n-2}{n-1}\right)
$$

The luminance and contrast can be assessed using the work descriptor in this method and can be shown by (a) DCab, (b) BCAD, (c) BCad in $d$ - $l$ scatter chart as shown in Fig. 4. However, because this method was expressed such as "very bright, very high contrast" has a lack of objectivity and practicality. As shown in the results below, word descriptor is hard to determine the status of image quality as the intuitive representation, and selection of a gamma value $(Y)$ to improve the image quality as the gamma correction is difficult.

To overcome these limitations, we propose a normalized numeric image descriptor to assess the luminance and contrast of the images. The proposed image descriptor uses each pixel's data as a weighted value of the probability density function. It is further defined by normalization for objective representation. The proposed numeric image descriptor can suggest an objective basis of the gamma value selection also, we propose a consistent normalization technique irrespective of image resolution and data representation bits. In particular, it is possible to use tone mapping to illumination changes and low illumination conditions because of its high dynamic range.

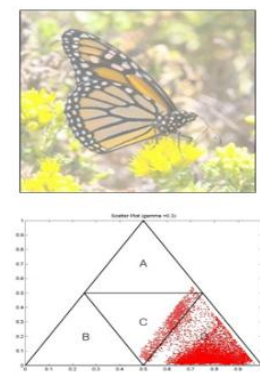

(a) gamma $=0.3$

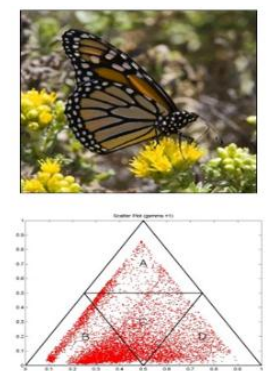

(b) gamma $=1$

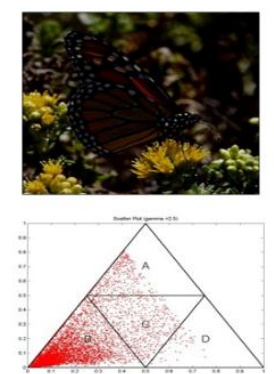

(c) gamma $=2.5$
Fig. 4. The methods by existing word descriptor 


\section{PROPOSED IMAGE QUALITY EVALUATION}

We are proposing an objective image quality evaluation $(O I Q E)$ method to evaluate the quality of the images. This method can improve the quality of the images under various non-ideal conditions such as low illumination and sudden illumination changes. The block schematic of the proposed method is as shown in Fig. 5. The input image which is in the RGB mode is converted into a YCbCr color model. The converted $\mathrm{YCbCr}$ color model runs the set block using $\mathrm{Y}$ value which has the luminance information. Then using a normalized numeric descriptor, the normalization of luminance $(\mathrm{NoL})$ and normalization of contrast $(\mathrm{NoC})$ is estimated. Finally, an objective image quality evaluation $(O I Q E)$ descriptor is used to generate the enhanced image using the information of normalized luminance and contrast.

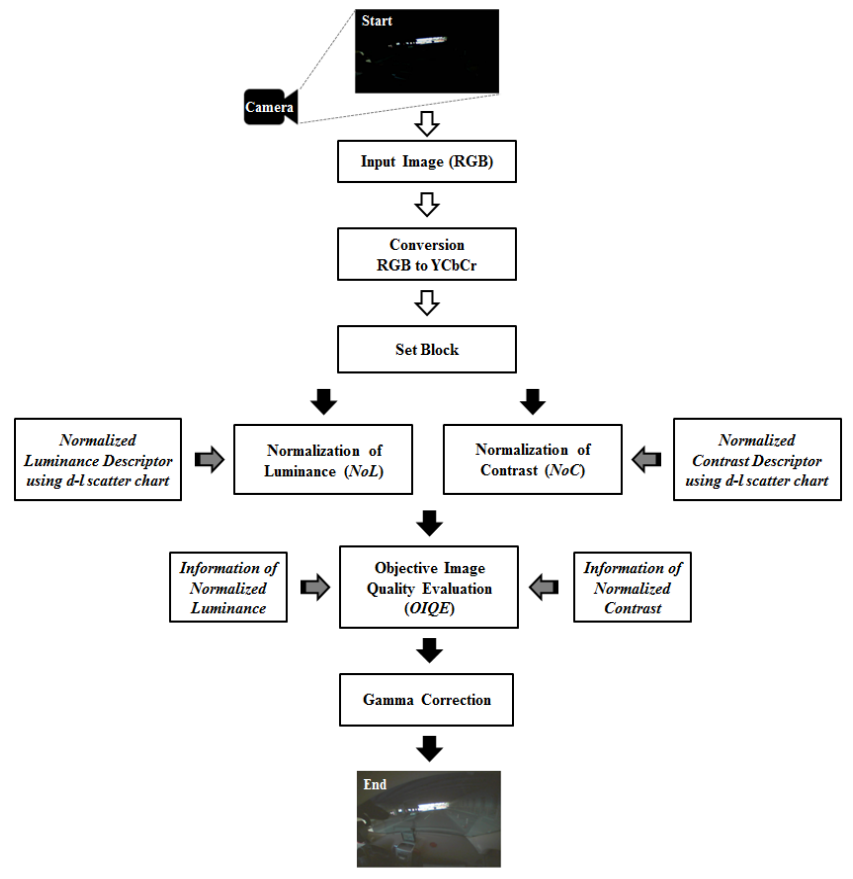

Fig. 5. Block diagram of a proposed method

\section{III.I Normalization of Luminance (NoL)}

The most basic quality evaluation of luminance and contrast is based on the histogram. The histogram of the gray image in range $([0, L-1])$ can be represented by equation (4).

$$
h\left(r_{k}\right)=n_{k}
$$

Where $r_{\mathrm{k}}$ is the $k$-th gray level and $n_{\mathrm{k}}$ is the number of pixels with $k$-th gray level. Since gray level can be used to weight the luminance of the images, luminance information (luminance descriptor, $L D$ ) can be expressed as equation (5).

$$
L D=\sum_{k=0}^{L-1}\left(r_{k} \cdot n_{k}\right)
$$

Since $L D$ depends on the resolution of the image, equation (5) needs to be normalized. The normalization can be obtained by dividing the image resolution from the value of $L D$. This is expressed in equation (6).

$$
N o L=\frac{1}{L N} \sum_{k=0}^{L-1} r_{k} \cdot n_{k}, 0 \leq N o L \leq 1
$$

Where, $L$ is the maximum value of the input image pixels (if the pixel width is 8 bits, the maximum value of $L$ is 255 ) and $N$ is the resolution size of the input image.

The proposed Normalization of Luminance $(\mathrm{NoL})$ has been applied to a "boat" image as shown in Fig. 6. The $d$ - $l$ scatter chart intuitively is possible to confirm with the naked eye. The gamma $(Y)$ value using NoL have something in common between $d-l$ scatter and $N o L$ descriptor.

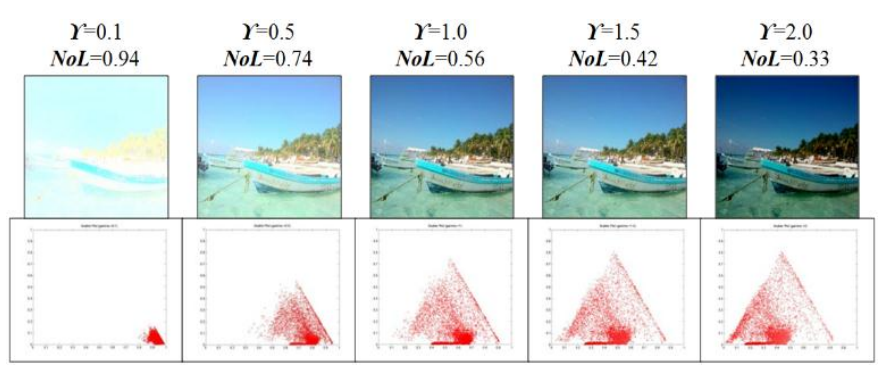

Fig. 6. The "Boat" image results of $N o L$ for each gamma value and its scattering chart

\section{III.II Normalization of Contrast (NoC)}

Since the contrast information is not included in $N o L$, it is required to consider the expression method. The difference between the maximum and minimum values of the pixels of the image represents the contrast information and this is shown in equation (7).

$$
c=r_{\max }-r_{\min }
$$

In order to obtain the contrast information (Contrast Descriptor ; $C D)$, histogram $\mathrm{h}\left(c_{l}\right)=b_{l}$ and the gray image in range $([0, L-1])$ is used. Where $c_{l}$ is the contrast of the $l$-th block. It has been calculated as $c_{l}=\mathrm{b}\left(r_{\max }-r_{\min }\right)_{l}$. The $b_{l}$ is the number of blocks of $c_{l}$ contrast value. In the same way, the value of $\mathrm{NoC}$ in equation (9) can be obtained through the normalization of equation (8).

$$
\begin{aligned}
C D & =\sum_{i=0}^{B-1} c_{l} \cdot b_{l} \\
N o C & =\frac{1}{L} \sum_{l=0}^{B-1} c_{l} \cdot b_{l}, 0 \leq N o C \leq 1
\end{aligned}
$$

It is verified using the "gravelly field" image as shown below. As shown in Fig. 7, the gamma value using $N o C$ have something in common between $d-l$ scatter and $N o C$ descriptor.

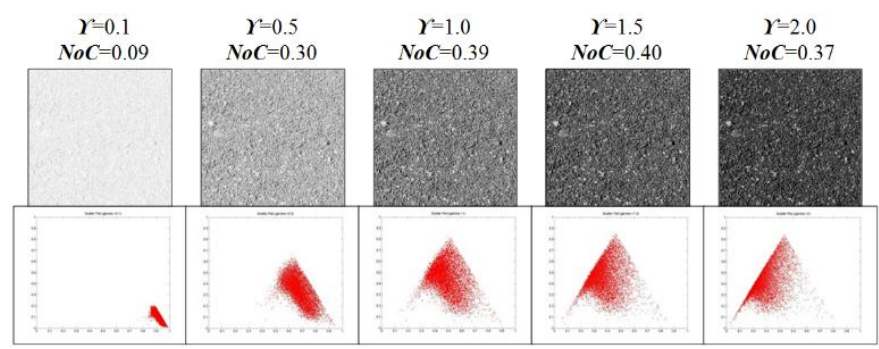

Fig. 7. The "Gravelly field" image results of $\mathrm{NoC}$ for each gamma value and its scattering chart

As a result of the equation (9), if NoC descriptor value approaches 1, the image is estimated to have high contrast and 
International Journal of Engineering Research and Technology. ISSN 0974-3154, Volume 13, Number 5 (2020), pp. 1057-1064

(C) International Research Publication House. https://dx.doi.org/10.37624/IJERT/13.5.2020.1057-1064

it will be estimated to have low contrast if $\mathrm{NoC}$ descriptor values approaches 0 .

\section{III.III Objective Image Quality Evaluation (OIQE)}

From the previous results, the normalized equation (10) of image quality concerning luminance and contrast is presented by arranging equations (6) and (9).

$$
O I Q E=(N o L, N o C)
$$

Therefore, it is possible to provide objective information about an image and at the same time, the ambiguity of conventional methods is eliminated too.

To prove this, we chose four sample images of "Airplane", "Monkey", "Butterfly", and "Gravelly field" where all the images have different resolution and frequency distribution. Each of the images has experimented while changing in units of 0.1 level using a gamma value of 0.1 to 3.0. The resolution of various images tested are from $200 \times 200,356 \times 356$ and $500 \times 500$, and it consists of the RGB color model of 24-bits. The images used in the experiment are separated as lowfrequency image, high frequency image and the difference frequency image. The experiment results compare d-l scatter and corrected image.

\section{Experiment 1}

The "Airplane" image shown in Fig. 8 consists of a lowfrequency distribution in $200 \times 200$ resolution. If the resultant image is very dark or very bright, then the contrast information is reduced. In the case of appropriate brightness, contrast information appears generally well. It has something in common between d-1 scatter and proposed objective image quality evaluation (OIQE). Also, Table 1 shows the characteristic curve for each descriptor in Fig. 8.

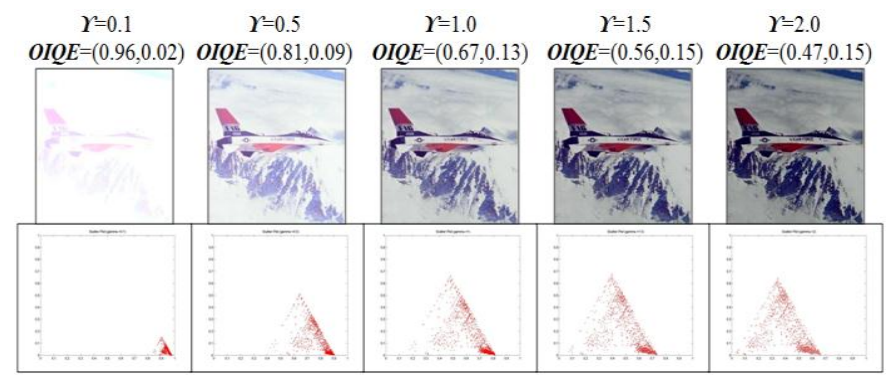

Fig. 8. The "Airplane" image results of $O I Q E$ for each gamma value and its scattering chart.

Table 1. Characteristic curve of "Airplane" image for each descriptor

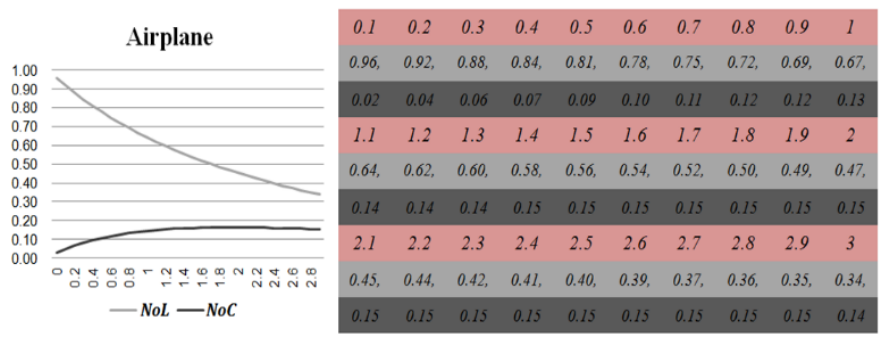

\section{(B) Experiment 2}

The "Monkey" image with a resolution of 200x200 which is tested is shown in Fig. 9 below. The low and the high frequency are distributed evenly.If the frequency components are very high or very low, the contrast information regardless of brightness is generally low. This result can be confirmed to have the same result between the naked eye and OIQE. Table 2 shows a characteristic curve for each descriptor in Fig. 9.

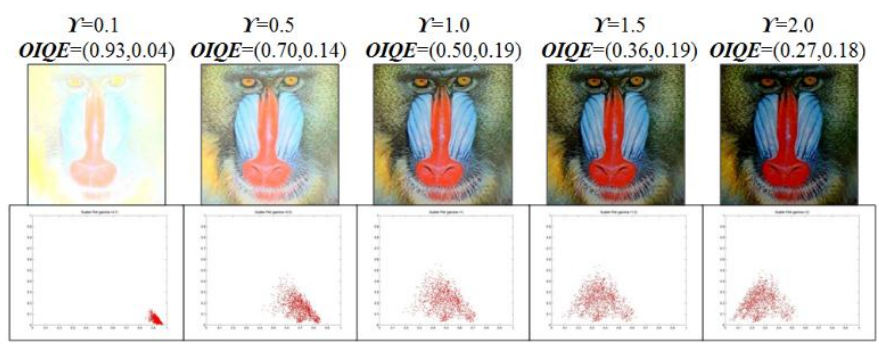

Fig. 9. The "Monkey" image results of $O I Q E$ for each gamma value and its scattering chart.

Table 2. Characteristic curve of "Monkey" image for each descriptor
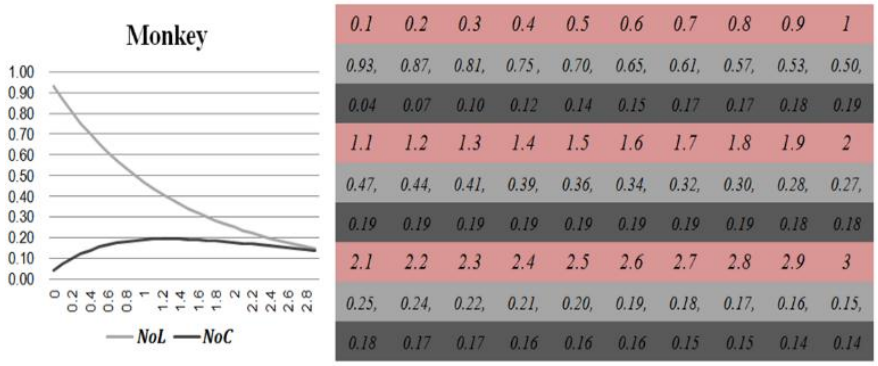

(C)

Experiment 3

The resolution of "Butterfly" image is $500 \times 500$ and the frequency was evenly distributed from low to high frequencies. The contrast information of the image is changed in accordance with the brightness of the image. As expected, the results of this experiment also correspond with the naked eye. This result can be confirmed by $d-l$ scatter as shown in Fig. 10. The numeric descriptor and characteristic curve are represented as shown in Table 3.

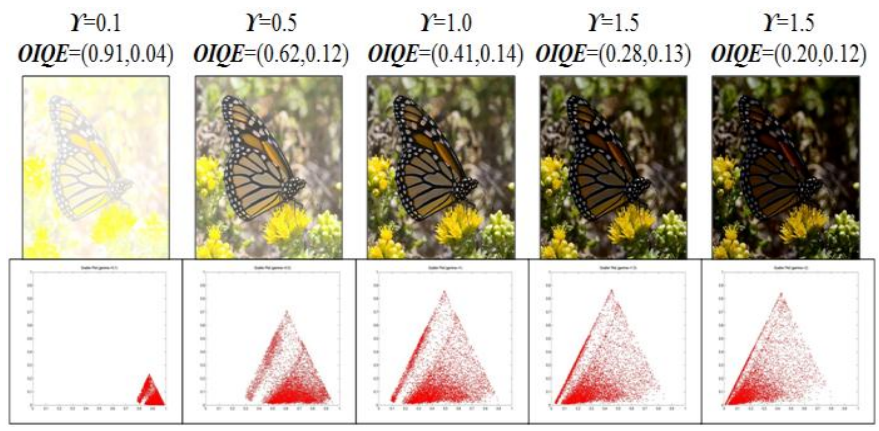

Fig. 10. The "Butterfly" image results of $O I Q E$ for each gamma value and its scattering chart. 
International Journal of Engineering Research and Technology. ISSN 0974-3154, Volume 13, Number 5 (2020), pp. 1057-1064

(C) International Research Publication House. https://dx.doi.org/10.37624/IJERT/13.5.2020.1057-1064

Table 3. Characteristic curve of "Butterfly" image for each descriptor
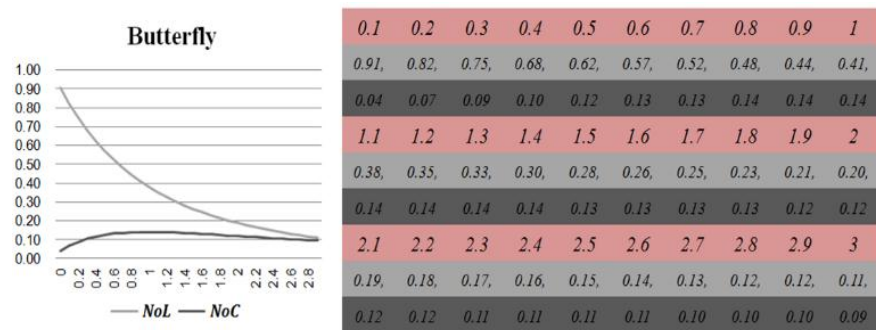

\section{(D)}

\section{Experiment 4}

The resolution of "Gravelly Field" image is $356 \times 356$ and the frequency distribution is distributed to high frequency in Fig. 11. Since the amplitude of frequency is large, in the case of a bright image the contrast information has a high value. If it is a dark image, the contrast information is reduced due to attenuated signal amplitude. But the contrast information is more. As a result, Fig. 11 and Table 4 can be compared.

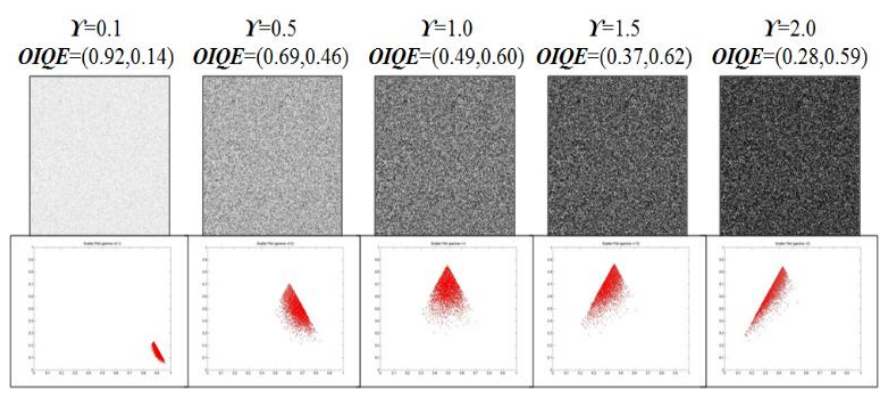

Fig. 11. The "Gravelly field" image results of OIQE for each gamma value and its scattering chart.

Table 4. Characteristic curve of "Gravelly field" image for each descriptor

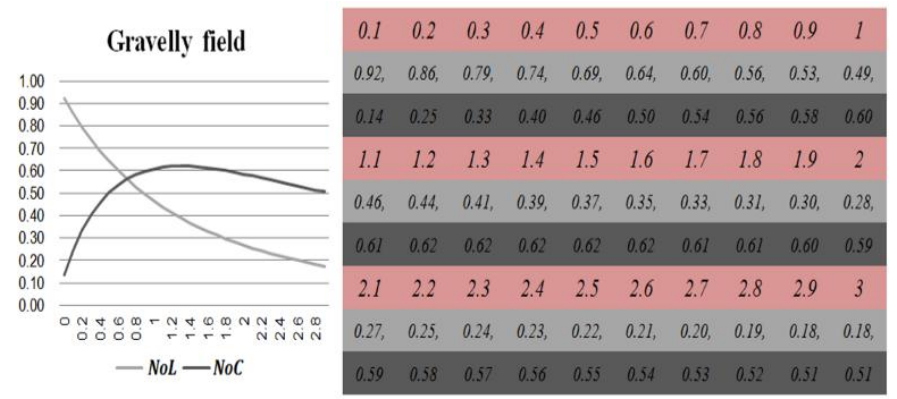

From the experimental results, when the gamma value of the image is linearly increased, the $O I Q E$ descriptor of each image can confirm the change. In the case of NoL descriptor, if it approaches 1, the brightness is more and when it approaches 0 the image is darker. In the case of $\mathrm{NoC}$ descriptor, a low value may results in very bright or very dark whereas a high value will display the image vividly.

\section{EXPRIMENTAL RESULTS AND ITS APPLICATION}

We have demonstrated improved restoration of images under non-ideal situations such as low illumination and sudden illumination changes of moving vehicles and other CCTV images. The experimental illustration is explained in the following section. The proposed technique combines various image processing methods. All the images tested were captured using CMOS image sensors. The system is implemented in an environment of Visual Studio 2010 and OpenCV 2.2, using the Intel (R) Core (TM)2 Quad Q8400 CPU $2.66 \mathrm{GHz}$ hardware. The video playback is possible with the support of GUI (Graphical User Interface). The gamma adjustment is possible with this proposed method. The original videos are shown on the left and the corrected gamma-adjusted videos are shown on the right.
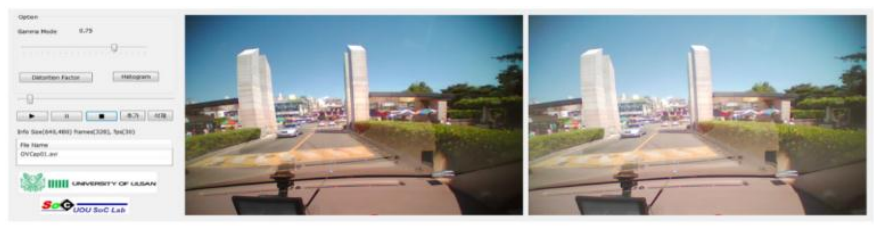

Fig. 12. Left: original images, Right: corrected images.

(Clip 1 : situation of high illumination)
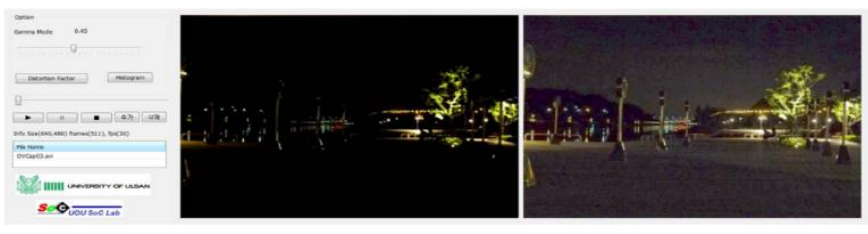

Fig. 13. Left: original images, Right: corrected images. (Clip 2 : situation of low illumination)

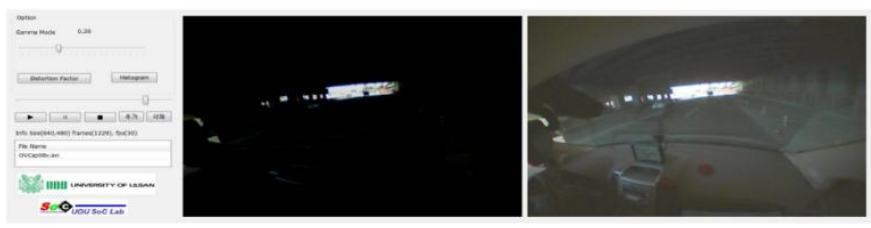

Fig. 14. Left: original images, Right: corrected images. (Clip 3 : situation of illumination change)

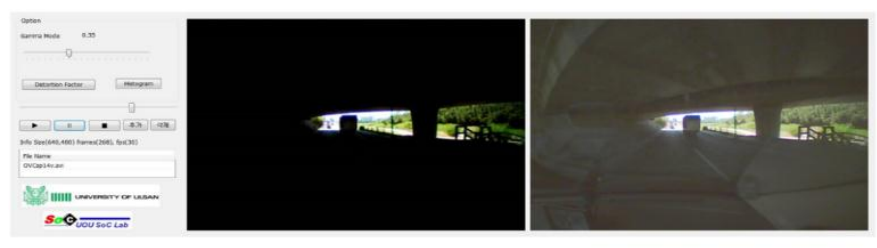

Fig. 15. Left: original images, Right: corrected images. (Clip 4 : situation of illumination change) 
International Journal of Engineering Research and Technology. ISSN 0974-3154, Volume 13, Number 5 (2020), pp. 1057-1064

(C) International Research Publication House. https://dx.doi.org/10.37624/IJERT/13.5.2020.1057-1064

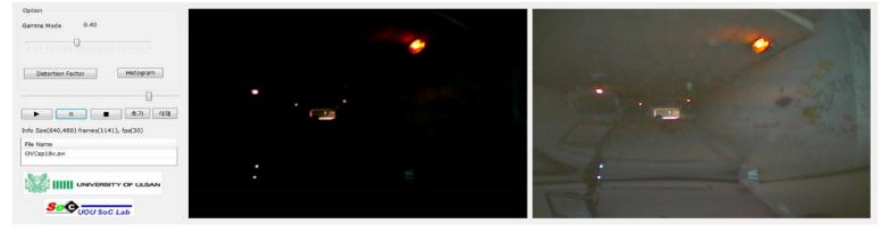

Fig. 16. Left: original images, Right: corrected images. (Clip 5 : situation of illumination change)

The original image of low illumination under overnight condition is difficult to ensure visibility as shown in Fig. 13. However, the right image in Fig. 13 shows the corrected results. The sky, land, mountain and structures are distinguished with the naked eye. Figs. 14 to Fig. 16 show the corrected results in the situation of a sudden illumination change when the vehicle passes through the tunnel and under a bridge.The next subsections show the figures for some applications that various algorithm have achieved through the combination of our proposed methods.

(A) Application 1-Lane Detection in Night Time
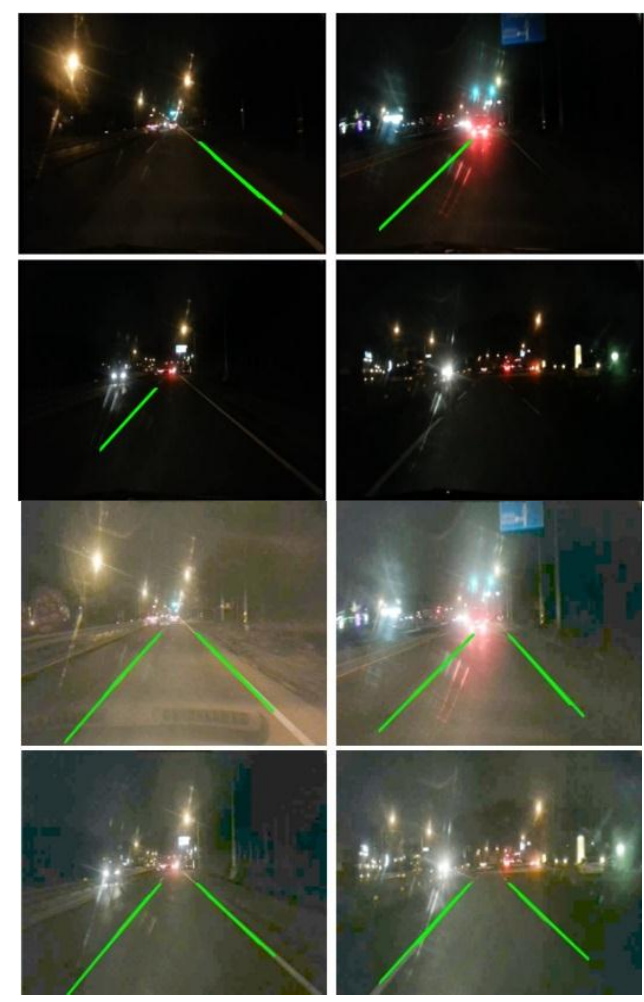

Fig. 17. Result of proposed method: (a) non-applied (b) applied

Each of Figs. 17 (a) and (b) shows the applied and nonapplied result for a proposed method on road condition during overnight low illumination conditions. The results are calculated and tabulated in table. 5. The detection rate has increased by $16 \%$ compared to conventional methods. Table 6 shows the comparison results between the existing state of the art method and the proposed method. The average detection rate of the existing method is $93.25 \%$ for the images of same resolution [11][12][13] whereas the average detection rate of the proposed method is over $99 \%$ including daytime, nighttime, and other non-ideal road conditions.

(B) Application 2-Number plate Detection from a moving vehicle
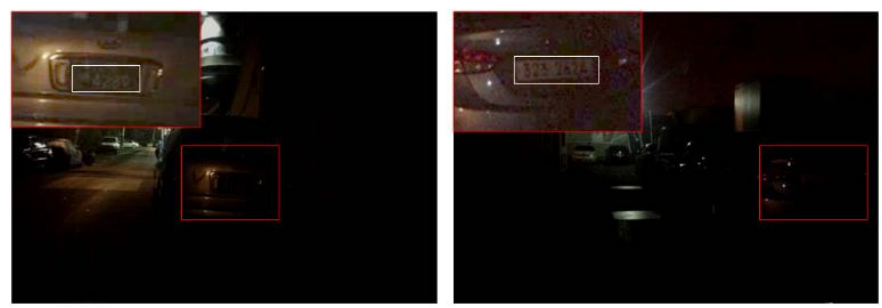

Fig. 18. The results of the number plate detection using the proposed method

Fig. 18 shows the result of number plate detection, corresponding to moving vehicles at night time and Table 7 displays the result. The detection rate has increased by $82 \%$ more than conventional methods.

(C) Application 3-Lens Distortion correction of the camera

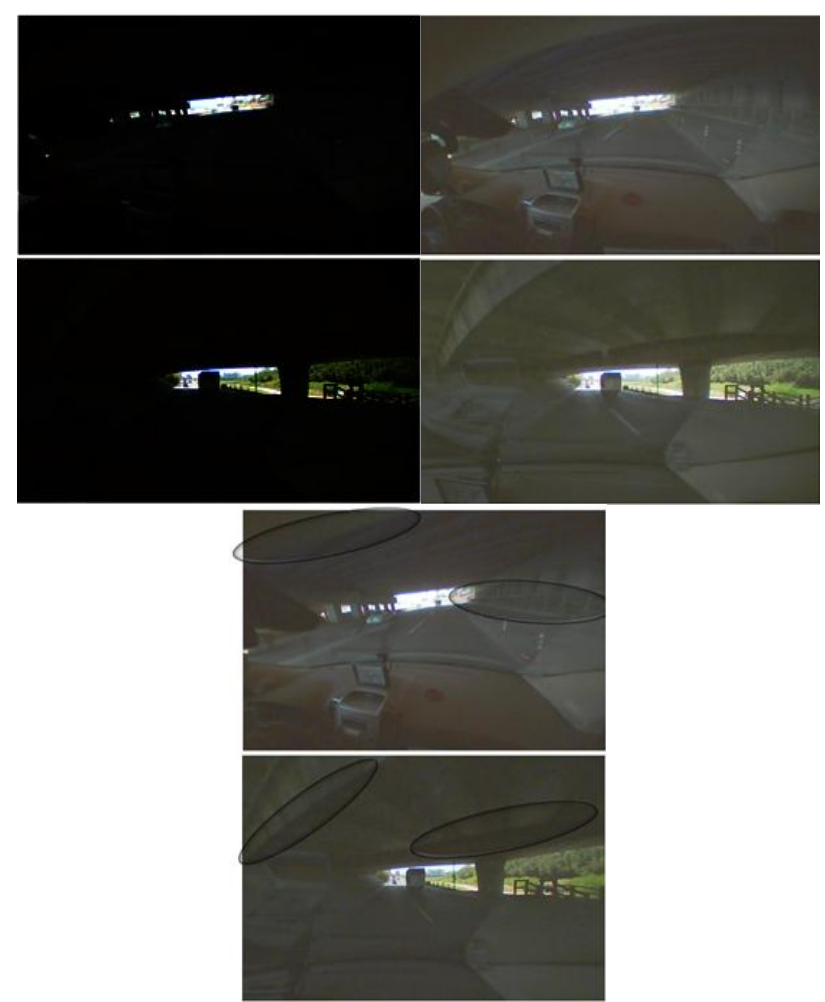

Fig. 19. The result combined of gamma and lens distortion correction

Each of Figs. 19 (a) and (b) shows a comparison result between gamma correction and additional lens distortion correction. The performance indicator for the integrated 
International Journal of Engineering Research and Technology. ISSN 0974-3154, Volume 13, Number 5 (2020), pp. 1057-1064

(C) International Research Publication House. https://dx.doi.org/10.37624/IJERT/13.5.2020.1057-1064

application of the gamma correction and lens distortion correction is tabulated in Table 8 .

Table 5. Comparison result of before and after for lane detection

\begin{tabular}{|l|c|c|c|c|}
\hline & $\begin{array}{l}\text { Total } \\
\text { Frame }\end{array}$ & $\begin{array}{l}\text { Detected } \\
\text { Frame }\end{array}$ & Frame/Sec & $\begin{array}{l}\text { Detection } \\
\text { Rate }\end{array}$ \\
\hline \hline $\begin{array}{l}\text { Before } \\
\text { (non- } \\
\text { applied) }\end{array}$ & 2760 & 2373 & 30.0 & $82.35 \%$ \\
\hline $\begin{array}{l}\text { After } \\
\text { (applied) }\end{array}$ & 2760 & 2730 & 30.0 & $98.9 \%$ \\
\hline
\end{tabular}

Table 6. Comparison result between existing and proposed method

\begin{tabular}{|l|l|l|l|l|}
\hline & {$[11]$} & {$[12]$} & {$[13]$} & Proposed \\
\hline \hline Total Frame & 13282 & 4172 & - & 9030 \\
\hline $\begin{array}{l}\text { Detected } \\
\text { Frame }\end{array}$ & 10984 & 4517 & - & 8955 \\
\hline Frame/Sec & 25.0 & - & 30.0 & 30 \\
\hline Resolution & VGA & VGA & VGA & VGA \\
\hline Correct Rate & $92.9 \%$ & $90.89 \%$ & $95.95 \%$ & $99 \%$ \\
\hline
\end{tabular}

Table 7. Comparison result of before and after for number plate detection

\begin{tabular}{|l|c|c|c|c|}
\hline & Resolution & $\begin{array}{l}\text { Total } \\
\text { Image }\end{array}$ & $\begin{array}{l}\text { Missed } \\
\text { Image }\end{array}$ & $\begin{array}{l}\text { Recognition } \\
\text { Rate }\end{array}$ \\
\hline \hline $\begin{array}{l}\text { Before } \\
\text { (non- } \\
\text { applied) }\end{array}$ & VGA & 60 & 52 & $13 \%$ \\
\hline $\begin{array}{l}\text { After } \\
\text { (applied) }\end{array}$ & VGA & 60 & 3 & $95 \%$ \\
\hline
\end{tabular}

Table 8. Performance indicators for application

\begin{tabular}{|l|l|l|l|l|}
\hline & Resolution & Frame/Sec & $\begin{array}{l}\text { Data } \\
\text { Speed }\end{array}$ & $\begin{array}{l}\text { Performance } \\
\text { time }\end{array}$ \\
\hline \hline Unit & pixel & fps & Kbps & sec \\
\hline $\begin{array}{l}\text { Numerical } \\
\text { data }\end{array}$ & VGA & 30 & 268 & 0.9 \\
\hline
\end{tabular}

\section{CONCLUSION}

The evaluation of the image quality related to the luminance and contrast must take precedence to improve the image quality for non-ideal conditions such as sudden illumination changes and low illumination conditions. In this paper, we proposed an objective image quality evaluation (OIQE) to overcome these non-ideal situations. The proposed image descriptor uses each pixel's data as a weighted value of the probability density function. It is further defined by normalization for objective representation. The normalization technique always can display images consistently irrespective of change in image resolution or change in the number of represented data bits. Also, it can be used in the adaptive gamma process since it suggests an objective basis of the gamma value selection. Tone mapping can be used to sudden illumination changes and low illumination conditions due to its high dynamic range. The proposed method can be incorporated with various application such as lane detection, number plate recognition, lens distortion correction etc. As a result, when this technique combined with existing applications and are used in harsh conditions (non-ideal), that cannot be easily taken with the camera lens, the performance and detection rate of the object can be enhanced.

\section{ACKNOWLEDGEMENTS}

This work was supported by the 2016 research fund of University of Ulsan, Ulsan, Republic of Korea

\section{REFERENCES}

[1] S. Marengo, C. Pepin, T. Goulet, and D. Houde.,1999, "Time-Gated Transillumination of Objects in Highly Scattering Media Using a Subpicosecond Optical Amplifier," IEEE Journal of Selected Topics in Quantum Electronics, vol. 5, no. 4, pp. 895-901.

[2] ChingSeong Tan, Gerald Seet, Andrzej Sluzek, DuoMin He.,2005, "A novel application of range-gated underwater laser imaging system (ULIS) in near-target turbid medium," Optics and Lasers in Engineering, vol. 43, no. 9, pp. 995-1009.

[3] John G. Walker, Peter C. Y. Chang, and Keith I. Hopcraft.,2000, "Visibility depth improvement in active polarization imaging in scattering media," Optical Society of America, vol. 39, no. 27, pp. 4933-4941.

[4] Yoav Y. Schechner, Srinivasa G. Narasimhan, and Shree K. Nayar,2003, "Polarization-based vision through haze," Optical Society of America, vol. 42, no. 3, pp. 511-525.

[5] Norman S. Kopeika and Joseph Bordogna, 1970, "Background Noise in Optical Communication Systems," in Proc. of IEEE, vol. 58, no. 10, pp. 1571 1577.

[6] John P. Oakley, and Brenda L. Satherley,1998, "Improving Image Quality in Poor Visibility Conditions Using a Physical Model for Contrast Degradation," IEEE Transactions on Image Processing, vol. 7, no. 2, pp. 167-179.

[7] Shree K. Nayar and Srinivasa G. Narasimhan,1999, "Vision in Bad Weather," in Proc. of IEEE International Conference on, vol. 2, pp. 820-827.

[8] Alfredo Restrepo (Palacios) and Giovanni Ramponi,2008, "Word Descriptors of Image Quality 
International Journal of Engineering Research and Technology. ISSN 0974-3154, Volume 13, Number 5 (2020), pp. 1057-1064

(C) International Research Publication House. https://dx.doi.org/10.37624/IJERT/13.5.2020.1057-1064

Based on Local Dispersion-versus-Location Distributions," 16th EUSIPCO, pp. 25-29.

[9] John P. Oakley and Hong Bu, 2007, "Correction of Simple Contrast Loss in Color Images," IEEE Transactions on Image Processing, vol. 16, pp. 511-522.

[10] J. Alex Stark,2000, “Adaptive Image Contrast Enhancement Using Generalizations of Histogram Equalization," IEEE Transactions on Image Processing, vol. 9, no. 5, pp. 511-522.

[11] Qingquan Li, Long Chen, Ming Li, Shih-Lung Shaw, and Andreas Nüchter, 2014, "A Sensor-Fusion Drivable-Region and Lane-Detection System for Autonomous Vehicle Navigation in Challenging Road Scenarios," IEEE Transactions on Vehicular Technology, vol. 63, no. 2, pp. 540-555.

[12] Mohamed Aly, 2008, "Real time Detection of Lane Markers in Urban Streets," IEEE Intelligent Vehicles Symposium, pp. 7-12.

[13] Amol Borkar, Monson Hayes and Mark T. Smith., 2012, "A Novel Lane Detection System With Efficient Ground Truth Generation," IEEE Transactions on Intelligent Transportation Systems, vol. 13, no. 1, pp. 365-374. 